\title{
EXIST perspective for Supergiant Fast X-ray Transients
}

\author{
Lara Sidoli* \\ INAF/IASF Milano Italy \\ E-mail: sidoli@iasf-milano.inaf.it
}

Vito Sguera

INAF/IASF Bologna Italy

E-mail: squeraliasfbo.inaf.it

\section{Angela Bazzano}

INAF/IASF Roma Italy

E-mail: angela.bazzano@iasf-roma.inaf.it

\section{Pietro Ubertini}

INAF/IASF Roma Italy

E-mail: pietro.ubertini@iasf-roma.inaf.it

\begin{abstract}
Supergiant Fast X-ray Transients (SFXTs) are one of the most intriguing (and unexpected) results of the INTEGRAL mission. They are a new class of High Mass X-ray Binaries involving about 20 sources to date, with 8 firmly identified SFXTs and many candidates. They are composed by a massive OB supergiant star as companion donor and a compact object. At least four SFXTs host a neutron star, because X-ray pulsations have been discovered, while for the others a black hole cannot be excluded. SFXTs display short X-ray outbursts (compared with Be/X-ray transients) characterized by fast flares on brief timescales of hours and large flux variability typically in the range 1,000-100,000. The INTEGRAL/IBIS sensitivity allowed to catch only the bright flares (peaking at $10^{36}-10^{37} \mathrm{erg} \mathrm{s}^{-1}$ ), without persistent or quiescent emission. The investigation of their properties, in particular the rapid variability time scales of their flaring activity, will greatly benefit from observations with the Energetic X-ray Imaging Survey Telescope (EXIST), with the possibility to perform a long term and continuous as possible monitoring of the hard X-ray sky.
\end{abstract}

The Extreme sky: Sampling the Universe above $10 \mathrm{keV}$

October 13-17 2009

Otranto (Lecce) Italy

${ }^{*}$ Speaker. 


\section{SFXTs properties}

In the last years a new class of fast X-ray transient sources, composed of an accreting compact object and an OB supergiant companion, has been discovered mainly by the IBIS instrument on board the INTEGRAL satellite: the so called Supergiant Fast X-ray Transients (SFXTs, Sguera et al. 2005). They spend most of the time in a low level X-ray activity with luminosities values in the range $10^{32}-10^{34} \mathrm{erg} \mathrm{s}^{-1}$, only occasionally undergoing bright $\left(\mathrm{L}_{x}=10^{36}-10^{37} \mathrm{erg} \mathrm{s}^{-1}\right)$ and fast flaring activity, part of outbursts with a duration of a few days. With their dynamic range of about $10^{3}-10^{5}$, SFXTs are among the most extreme examples of variability in the X-ray/soft gamma-ray sky. Their apparently short duty cycle as well as transitory nature are the main reasons why they escaped detection in the previous forty years of X-ray observations. In fact, since SFXTs occur at unpredictable locations and times, it is very difficult to detect and discover them using traditional narrow field X-ray instruments. On the contrary, detectors having a sufficiently wide field of view, such as IBIS, are particularly suited to detect SFXTs, allowing a greater chance of serendipitously detecting a short duration $\mathrm{X}$-ray event.

The physical mechanism driving the peculiar transient X-ray emission from SFXTs is unclear and still highly debated: some models involve the structure of the supergiant wind (likely clumpy, in a spherical or non spherical geometry; in't Zand 2005; Negueruela et al. 2006; Sidoli et al. 2007; Ducci et al. 2009) and the orbital properties (wide separation with eccentric or circular orbit), while others involve the properties of the neutron star and invoke very low magnetic field values $\left(B<10^{10} \mathrm{G}\right.$; Sguera et al. 2009a) or alternatively very high $\left(\mathrm{B}>10^{14} \mathrm{G}\right.$, magnetars; Bozzo et al. 2008). The picture is still highly unclear from the observational point of view: no cyclotron lines have been detected to date in the SFXTs spectra, thus the strength of the neutron star magnetic field is unknown. Only in the SFXT IGR J18483-0311 a hint of a cyclotron emission line (which however needs confirmation) at $3.3 \mathrm{keV}$ has been found in the $X M M-N e w t o n$ spectrum, translating into a magnetic field of a few $\mathrm{B} \sim 10^{11} \mathrm{G}$ (Sguera et al. 2009b).

Periodicities, likely orbital, have been measured in five systems, spanning from only 3.3 days (IGRJ16479-4514; Jain et al. 2009) to 165 days (in IGRJ11215-5952; Sidoli et al. 2006, 2007; Romano et al. 2009a). Especially the shortest orbital period is puzzling, because it is even shorter than what is observed in several persistent HMXBs. Indeed, the link between HMXBs displaying persistent X-ray emission and SFXTs, is still unclear, and needs an in-depth investigation.

Even the SFXTs duty cycle seems to be quite different among the members of the class (Romano et al. 2009b). Thus a unified picture of the actual mechanism driving the outburst emission is still lacking, also in comparison with persistent HMXBs hosting a similar donor star.

\section{SFXTs science with EXIST}

The Energetic X-ray Imaging Survey Telescope (EXIST) will be particularly suited to the detection and subsequent study of new or already known Supergiant Fast X-ray Transient sources, since it will provide a very powerful combination of very large field of view (FOV), sensitivity and angular resolution, wide energy range coverage and accurate source positioning.

EXIST, with its hard X-ray imaging (5-600 keV) all-sky deep survey and large improved limiting sensitivity, will allow to explore the transient X-ray Universe to get a clearer picture of 


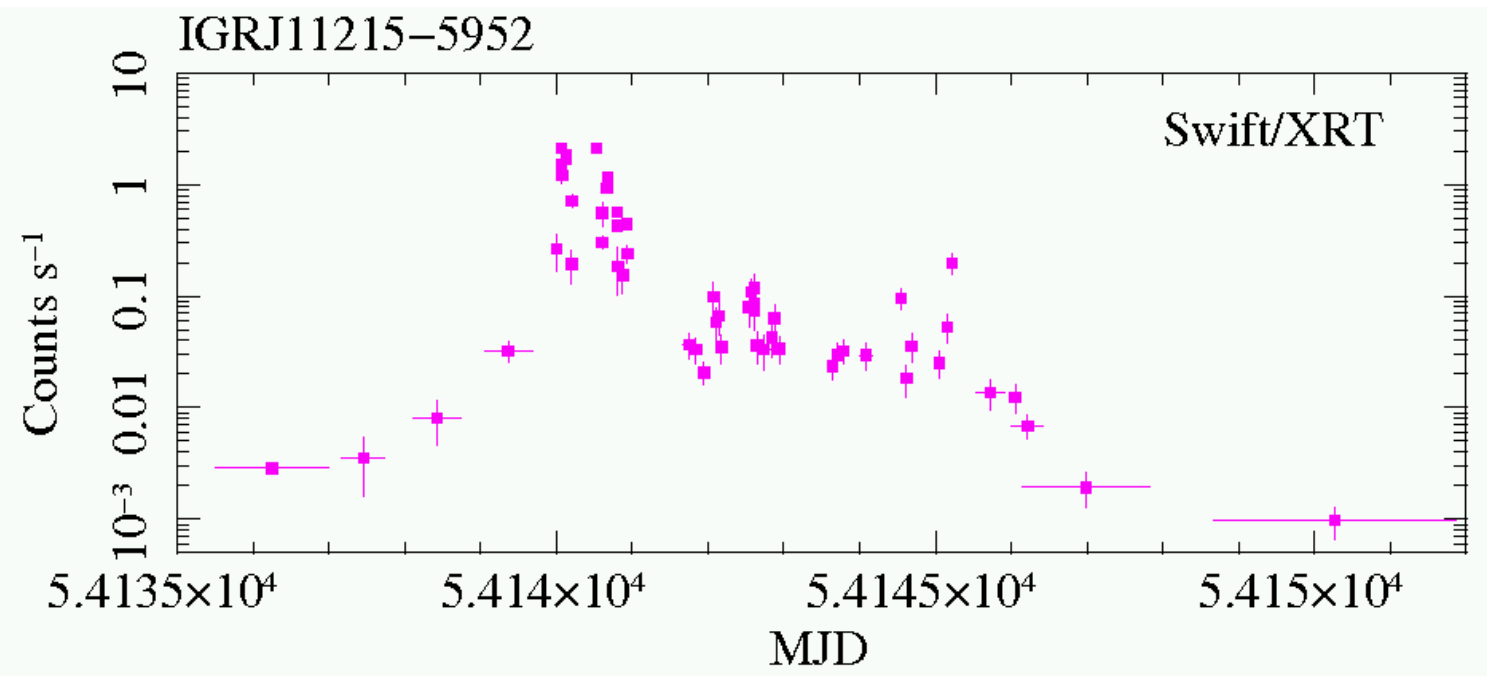

Figure 1: Swift/XRT lightcurve of the SFXT IGRJ11215-5952, during one of its periodic outbursts, in 2007 February

this new class of puzzling X-ray transients. A complete census of the number of fast transients is essential to enlarge the sample and possibly discover different behaviors and SFXTs sub-classes. A long term and continuous as possible X-ray monitoring of SFXTs is crucial to (1)-properly obtain the duty cycle, (2)-to investigate their orbital properties (separation, orbital period, eccentricity) which are unknown for the great majority of SFXTs and search for periodicities predicted by models, (3)-to completely cover the whole outburst activity (now possible only for SFXTs with periodically recurrent X-ray flaring activity, see Figure 1), (4)-to search for cyclotron lines in the high energy spectra which could provide the first firm measurement of the neutron star magnetic field. EXIST observations will help in shedding light also to the link between SFXTs and classical HMXBs which are persistent X-ray emitters. We estimate that EXIST, given its sensitivity about at least one order of magnitude higher than IBIS, will discover hundreds new SFXTs on the Galactic plane during one year scanning.

\section{References}

[1] Bozzo, E., Falanga, M., Stella, L., 2008, ApJ, 683, 1031

[2] in't Zand, J.J.M., 2005, $A \& A$, 441, L1

[3] Jain, C, Paul, B, Dutta, A., 2009, MNRAS, 397, L11

[4] Negueruela, I., Smith, D.M., Reig, P., et al. 2006, in ESA Sp. Pub., ed. A.Wilson, Vol. 604, 165-170

[5] Romano, P., Sidoli, L., Cusumano, G., et al., 2009a, ApJ, 696, 2068

[6] Romano, P., Sidoli, L., Cusumano, G., et al., 2009b, MNRAS, 399, 2021

[7] Sguera, V., Barlow, E.J., Bird, A.J., et al. 2005, $A \& A, 4$ 444, 221

[8] Sguera, V., Romero, G. E., Bazzano, A., et al. 2009, ApJ, 697, 1194

[9] Sguera, V., Ducci, L., Sidoli, L., Bazzano, A., Bassani, L., 2009, MNRAS in press, (arXiv 0912.1730) 
[10] Sidoli, L., Paizis, A., \& Mereghetti, S., 2006, A\&A, 450, L9

[11] Sidoli, L., Romano, P., Mereghetti, S., et al., 2007, A\&A, 476, 1307 